\section{Decomposition of Multidimensional Filters}

\section{A. N. VENETSANOPOULOS AND B. G. MERTZIOS}

\begin{abstract}
A theorem is proposed for the exact expansion of a general $m$-dimensional rational function in terms of order one, each one of which is a function of each of the $m$ variables. This decomposition can be used for the implementation of a general, linear IIR $m$-dimensional filter, with great modularity and parellelism in its structure.
\end{abstract}

\section{INTRODUCTION}

The fundamental theorem of algebra, which results in the factorization of $1-D$ polynomials, is a very important tool for the design and implementation of 1-D filters. The lack of a corresponding theorem in the $m$-dimensional case is a serious problem in the generalization of many filtering techniques [1]-[3]. In this paper, a decomposition theorem is proposed, which applies to $m$-dimensional polynomials and rational functions. The motivation behind this theorem is to provide a general implementation method with great modularity and parallelism applicable to $\mathrm{m}$ dimensional filters.

\section{Decomposition of a General $m$-Dimensional RATIONAL FUNCTION}

\section{Theorem:}

The $m$-dimensional rational function with real coefficients of the general form

$$
H(Z)=\frac{q(Z)}{p(\boldsymbol{Z})}=\frac{\sum_{k_{1}=0}^{n_{1}} \sum_{k_{2}=0}^{n_{2}} \ldots \sum_{k_{m}=0}^{n_{m}} q_{k_{1}, k_{2}, \ldots k_{m}} z_{1}^{k_{1}} z_{2}^{k_{2}}, \ldots, z_{m}^{k_{m}}}{\sum_{k_{1}=0}^{\hat{n}_{1}} \sum_{k_{2}=0}^{\hat{n}_{2}} \ldots \sum_{k_{m}=0}^{\hat{n}_{m}} p_{k_{1}, k_{2}, \ldots, k_{m}} z_{1}^{k_{1}} z_{2}^{k_{2}}, \ldots, z_{m}^{k_{m}}}
$$

can be expressed in terms of monomials of the form $\left(z_{i}-c_{i j}\right)$, $i=1,2, \cdots, m$, where $Z=\left\{z_{i} ; i=1,2, \cdots, m\right\}$ and $c_{i j}, i=1,2, \cdots$, are constants. Each factor of the denominator with $t$ variables requires feedback, with $t \leqslant m$ variables, for its implementation.

\section{Proof: (a) Numerator}

The polynomial $q(\boldsymbol{Z})$ can be written in the form

$$
\begin{aligned}
q(\boldsymbol{Z}) & =\sum_{k_{1}=0}^{n_{1}} z_{1}^{k_{1}}\left[\sum_{k_{2}=0}^{n_{2}} \cdots \sum_{k_{m}=0}^{n_{k}} q_{k_{1}, k_{2}, \cdots, k_{m}} z_{2}^{k_{2}} \cdots z_{m}^{k_{m}}\right] \\
& =\sum_{k_{1}=0}^{n_{1}} z_{1}^{k_{1}} \cdot a_{k_{1}\left(z_{2} \cdots z_{m}\right)}=\boldsymbol{Z}_{1}^{T} \cdot \boldsymbol{A}\left(z_{2}, \cdots, z_{m}\right)
\end{aligned}
$$

where

$$
\begin{aligned}
Z_{1} & =\left[\begin{array}{c}
1 \\
z_{1} \\
\vdots \\
n_{1} \\
z_{1}
\end{array}\right] \\
\boldsymbol{A}\left(z_{2} \cdots z_{m}\right) & =\left[\begin{array}{c}
a_{0}\left(z_{2}, \cdots, z_{m}\right) \\
a_{1}\left(z_{2}, \cdots, z_{m}\right) \\
\vdots \\
a_{n_{1}}\left(z_{2}, \cdots, z_{m}\right)
\end{array}\right]
\end{aligned}
$$

Manuscript received October 4, 1983.

A. N. Venetsanopoulos is with the Department of Electrical Engineering, University of Toronto, Toronto, Ont., Canada.

B. G. Mertzios is with the Department of Electrical Engineering, Democritus University of Thrace, Xanthi, Greece.
$A\left(z_{2}, \cdots, z_{m}\right)$ can be written as

$$
\boldsymbol{A}\left(z_{2}, \cdots, z_{m}\right)=\boldsymbol{R}_{1} \boldsymbol{S}_{1}\left(z_{2}, \cdots, z_{m}\right)
$$

where the matrix $\boldsymbol{R}_{1}$ is chosen to be a $\left(n_{1}+1\right) \times\left(n_{1}+1\right)$ nonsingular matrix, $S_{1}\left(z_{2}, \cdots, z_{m}\right)$ can be determined from (4) and the polynomial $q(Z)$ can be written

$$
q(Z)=\left[\boldsymbol{Z}_{1}^{T} \boldsymbol{R}_{1}\right]\left[\boldsymbol{R}_{1}^{-1} \boldsymbol{A}\left(z_{2}, \cdots, z_{m}\right)\right] .
$$

It can be readily seen that

$$
\boldsymbol{Z}_{1}^{T} \boldsymbol{R}_{1}=\left[r_{0}^{1}\left(z_{1}\right), r_{1}^{1}\left(z_{1}\right), \cdots, r_{i}^{1}\left(z_{1}\right), \cdots, r_{n_{1}}^{1}\left(z_{1}\right)\right]
$$

where

$$
r_{i}^{1}\left(z_{i}\right)=\sum_{j=0}^{n_{1}} r_{j i}^{1} z_{i}^{j}
$$

and

$$
R_{1}^{-1} A\left(z_{2}, \cdots, z_{m}\right)=\left[\begin{array}{c}
b_{0}\left(z_{2}, \cdots, z_{m}\right) \\
b_{1}\left(z_{2}, \cdots, z_{m}\right) \\
\vdots \\
b_{n_{1}}\left(z_{2}, \cdots, z_{m}\right)
\end{array}\right]
$$

hence

$$
q(Z)=\sum_{i=0}^{n_{1}} r_{i}^{1}\left(z_{1}\right) b_{i}\left(z_{2}, \cdots, z_{m}\right) .
$$

Continuing in the same way we now expand the polynomials $b_{i}\left(z_{2}, \cdots, z_{m}\right)$.

After applying this procedure a total of $m-1$ times we arrive to the following expression:

$$
\begin{gathered}
q(Z)=\sum_{k_{1}=0}^{n_{1}} \sum_{k_{2}=0}^{n_{2}} \cdots \sum_{k_{m-1}=0}^{n_{m-1}} r_{k_{1}}^{1}\left(z_{1}\right) r_{k_{2}}^{2}\left(z_{2}\right) \cdots \\
r_{k_{m-1}}^{m-1}\left(z_{m-1}\right) r_{k_{m-1}}^{m}\left(z_{m}\right)
\end{gathered}
$$

where $r_{k_{i}}^{1}\left(z_{i}\right), i=1, \cdots, m-1, k_{i}=0, \cdots, n_{i}$, are polynomials of order $n_{i}$. Each of these polynomials can be written as a product of monomials of order one, in each of the $m$ variables. Those monomials, which have complex parameters $c_{i j}$, can be combined to form second order terms with real coefficients.

(b) Denominator

Let the denominator polynomial $p(Z)$ be reducible to a product of lower order polynomials, i.e., the denominator can be written as

$$
p(Z)=\prod_{i=1}^{v} p_{i}
$$

where $p_{i}$ are irreducible polynomials containing $t_{i} \leqslant m$ of the variables $z_{1}, z_{2}, \cdots, z_{m}$. Writing the factor $p_{i}$ as $p_{i}=c_{i}+p_{i o}$, where $c_{i}$ is a constant and the polynomial $p_{i o}$ does not contain a constant term, the all-pole function is written as

$$
\begin{aligned}
H_{D}(Z) & =\frac{1}{p(Z)}=\prod_{i=1}^{\nu} \frac{1}{c_{i}+p_{i o}} \\
& =\prod_{i=1}^{\nu} \frac{1 / c_{i}}{1+\left(1 / c_{i}\right) p_{i o}}=\prod_{i=1}^{\nu} H_{D_{i}} .
\end{aligned}
$$

As it is seen (11), each factor $H_{D_{i}}$ can be realized by feedback containing $t_{i}$ variables. Subsequently, applying the same technique, outlined for the numerator term, $p_{i o}$ can be written in the form of (9). 


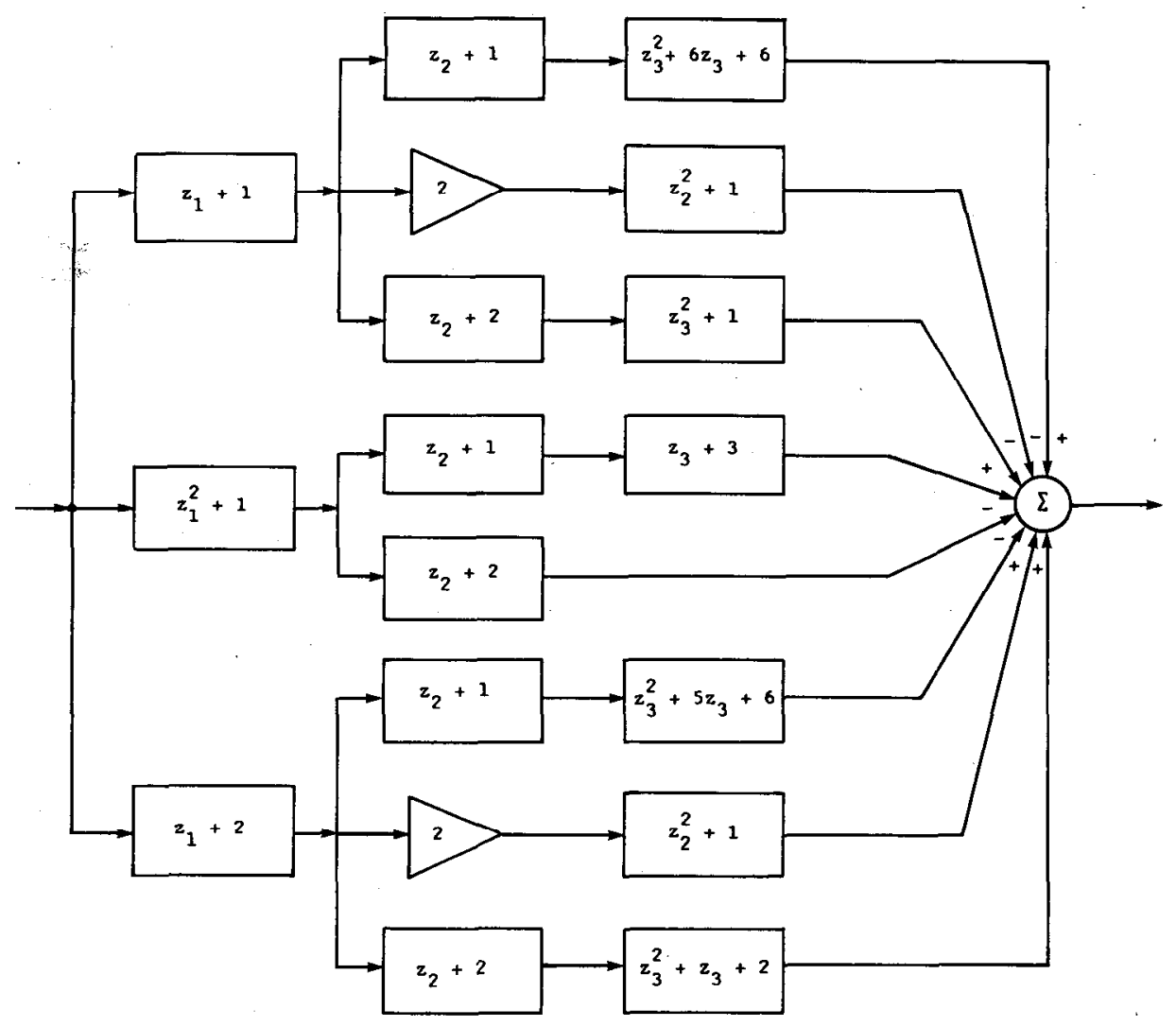

Fig. 1.

(c) Necessity of feedback containing $t$ variables

Let the polynomial $p(Z)$, be of the form (10). Assume that the factor $H_{L_{i}}$, for an all-pole function $H_{D}(Z)$ can be represented by a general lattice form without feedback, which contains the $t_{i} \leqslant m$ variables (those of the polynomial $p_{i}$ ). In such a case $I_{D_{i}}$ can be expressed in the form of (12).

$$
H_{D_{i}}=\frac{1}{p_{i}}=\sum_{i=1}^{L} \prod_{j=1}^{M} H_{i j}
$$

where $H_{i j}$ are factors, each one of which is a function of $t_{i}-1$ variables, at most. After combining all these terms, by creating a common denominator, we obtain a rational function with a factored denominator, which is a product of lower order polynomials, each one of which is a function of $t_{i}-1$ variables at most, and in the general case an irreducible numerator. Clearly it is seen from (12) that this common factored denominator equals to the product of the irreducible polynomial $p_{i}$, with the irreducible numerator. Since the denominator consists of lower order polynomials, each one of which has less than $t_{i}$ variables, (12) can not hold. Therefore we conclude that the implementation of $H_{D_{i}}$ requires a feedback loop, containing the $t_{i}$ variables of $p_{i}$. Q.E.D.

\section{EXAMPLE}

Consider the filter described by the transfer function

$$
H(Z)=\frac{q(Z)}{p(Z)}
$$

where

$$
\begin{aligned}
q(Z)= & z_{1}^{2} z_{2} z_{3}+2 z_{1}^{2} z_{2}+z_{1}^{2} z_{3}+2 z_{1} z_{2} z_{3}+z_{1} z_{2}+3 z_{1} z_{3} \\
& -z_{2} z_{3}+z_{1}^{2}+2 z_{2}^{2}+z_{3}^{2}+2 z_{1}-z_{2}+z_{3}+3 \\
p(Z)= & p_{1}\left(z_{1}, z_{2}\right) p_{2}\left(z_{1}, z_{2}, z_{3}\right) \\
= & \left(z_{1} z_{2}+z_{1}+z_{2}+2\right)\left(z_{1} z_{2} z_{3}+2 z_{1} z_{2}+z_{1} z_{3}\right. \\
& \left.-z_{2} z_{3}+z_{1}-z_{2}+z_{3}+1\right) .
\end{aligned}
$$

Choose

$$
Q=\left[\begin{array}{lll}
1 & 1 & 2 \\
1 & 0 & 1 \\
0 & 1 & 0
\end{array}\right]
$$

for the decomposition of both factors of the numerator. After carrying out the steps of the proposed algorithm, we obtain the configuration given in Fig. 1.

For the implementation of the denominator we consider separately the factors $p_{1}\left(z_{1}, z_{2}\right)$ and $p_{2}\left(z_{1}, z_{2}, z_{3}\right)$ of the denominator. Both of these factors have a constant term and, therefore, they can be implemented by realizable implementations. To this end, for the first factor we have

$$
\begin{aligned}
p_{1}\left(z_{1}, z_{2}\right) & =c_{1}+p_{01}\left(z_{1}, z_{2}\right) \\
& =2+\left(z_{1} z_{2}+z_{1}+z_{2}\right) .
\end{aligned}
$$

The polynomial $p_{01}\left(z_{1}, z_{2}\right)$ can be written as

$$
p_{01}\left(z_{1}, z_{2}\right)=\left[1, z_{1}\right]\left[\begin{array}{c}
z_{2} \\
1+z_{2}
\end{array}\right] \text {. }
$$

For the decomposition we choose the matrix $Q_{1}$ to be

$$
Q_{1}=\left[\begin{array}{ll}
1 & 0 \\
1 & 1
\end{array}\right]
$$

Then the polynomial $p_{01}\left(z_{1}, z_{2}\right)$ is written as

$$
p_{01}\left(z_{1}, z_{2}\right)=\left[z_{1}+1, z_{1}\right]\left[z_{2}, 1\right]^{T} \text {. }
$$

For the second factor $p_{2}\left(z_{1}, z_{2}, z_{3}\right)$ and using the same matrix $Q_{1}$ for all stages of its decomposition we have

$$
\begin{aligned}
p_{2}\left(z_{1}, z_{2}, z_{3}\right)= & c_{2}+p_{02}\left(z_{1}, z_{2}, z_{3}\right) \\
= & 1+\left(z_{1} z_{2} z_{3}+2 z_{1} z_{2}+z_{1} z_{3}-z_{2} z_{3}\right. \\
& \left.+z_{1}-z_{2}+z_{3}\right)
\end{aligned}
$$

where

$$
p_{02}\left(z_{1}, z_{2}, z_{3}\right)=\left[1, z_{1}\right]\left[\begin{array}{c}
-z_{2} z_{3}-z_{2}+z_{3} \\
z_{2} z_{3}+2 z_{2}+z_{3}+1
\end{array}\right] \text {. }
$$




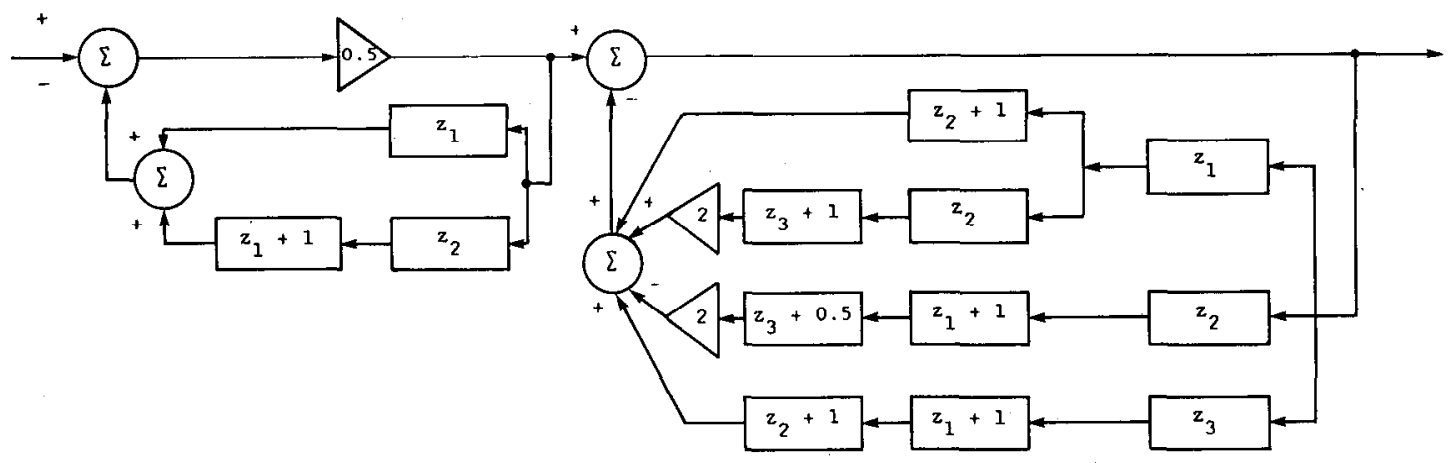

Fig. 2.

After carrying out all the steps of the proposed algorithm, we arrive in the implementation of the denominator given in Fig. 2.

\section{COMMENTS}

A gencral decomposition thcorem of a rational function of $m$ variables, in terms of functions of one variable only, leads to implementations of $m$-dimensional filters with great modularity and parallelism. Many different implementations can be achieved, depending on the choice of the matrices $\boldsymbol{R}_{i}$. These matrices can be chosen to achieve filter realizability, easy stability testing or optimum filter implementation. Such considerations have been already reported in the 2-D case [4], [5]. Their extension to the $m-D$ case is now in progress.

\section{REFERENCES}

[1] T. S. Huang. W. F. Schreiber, and O. I. Tretiak, "Image processing," Proc. IEEE, vol. 29, pp. 1586-1609, 1971.

[2] R. Eising, "Separability of 2-D transfer matrices," IEEE Trans. Automat. Contr. vol. AC-24, pp. 508-510, 1979.

[3] M. Morf, B. C. Levy, and S. Y. Kung, "2-D polynomial matrices, factorization and comprimeness," Proc. IEEE, vol. 65, pp. 861-872, 1977.

[4] A. N. Venetsanopoulos and B. G. Mertzios, "General decomposition of two-dimensional filters," Proc. oth European Conf. Circuit Theory and Design (ECCTD'83), (Stuttgart, FRG), pp. 444-446, Sept. 6-8, 1983.

[5] B. G. Mertzios and A. N. Venetsanopoulos "Noise properties of twodimensional IIR digital filters implemented by the decomposition theorem." in Proc. 2nd European Signal Processing Conf., (EUSIPCO'83), (Erlangen, FRG), Sept. 12-16, 1983.

\section{A Stability New Test for Linear Discrete Systems in a Table Form}

Y. BISTRITZ

Abstract - A new stability testing table is presented for discrete linear system. The table is similar to the Routh table in size, amount of computation as well as other features. The new table requires for a discrete system polynomials about half the number of entries and arithmetic operations that is needed in the previous Jury-Marden criterion in tabular form.

\section{The Stability Criterion}

Assume a real polynomial of degree $n$

$$
D(z)=d_{0} z^{n}+d_{1} z^{n-1}+\cdots+d_{n}, \quad d_{0}>0 .
$$

Define its reciprocated polynomial $D^{*}(z)$ as $z^{n} D\left(z^{-1}\right)$, that is $D^{*}(z)$ denotes the polynomial

$$
D^{*}(z)=d_{n} z^{n}+d_{n-1} z^{n-1}+\cdots+d_{0} .
$$

The polynomial $D(z)$ can be written as the sum

$$
D(z)=\frac{1}{2} S(z)+\frac{1}{2} A(z) .
$$

In the above $S(z)$ and $A(z)$ are the symmetric and antisymmetric polynomials, respectively, defined by

$$
\begin{aligned}
& S(z)=D(z)+D^{*}(z) \\
& A(z)=D(z)-D^{*}(z)
\end{aligned}
$$

where a general polynomial

$$
P(z)=\sum_{i=0}^{n} p_{i} z^{i}
$$

is called symmetric if

$$
P^{*}(z)=P(z) \quad p_{i}=p_{n-i}, \quad i=0,1, \cdots
$$

and it is called antisymmetric if

$$
P^{*}(z)=-P(z) \quad p_{i}=-p_{n-i}, \quad i=0,1, \cdots .
$$

Necessary and sufficient conditions for the polynomial $D(z)$ to have all its zeros inside the unit circle are imposed on the following table:

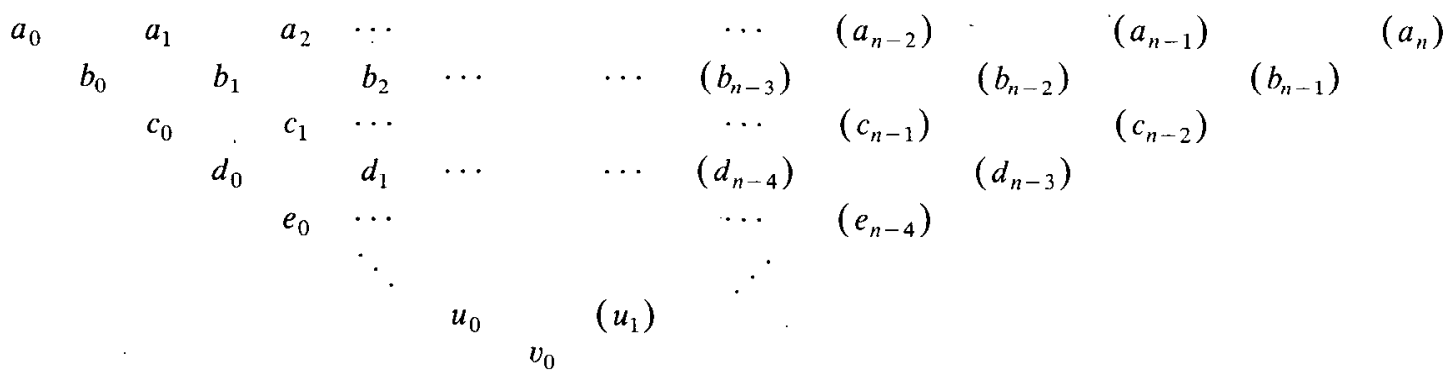

Manuscript received September 30, 1983.

The author is with the Department of Electrical Engineering, School of Engineering, Tel Aviv University, Ramat Aviv 69978, Israel. that is constructed as follows:

(i) The first two rows are 